\title{
A Global Approach for Medical Image Denoising via Sparse Representation
}

\author{
Yi Guo, Hanchao Chai, Yuanyuan Wang* \\ Department of Electronic Engineering, Fudan University, Shanghai, China. \\ * Corresponding author. Tel.: +86-21-65642756; email: yywang@fudan.edu.cn. \\ Manuscript submitted October 15, 2014; accepted December 20, 2014. \\ doi: 10.17706/ijbbb.2015.5.1.26-35
}

\begin{abstract}
In this paper, a novel global noise reduction approach based on the sparse representation and nonlocal means algorithm is proposed to enhance the image qualities of various medical imaging modalities, including ultrasound images and magnetic resonance images. By using an overcomplete dictionary, a medical image is decomposed into a sparsest coefficients matrix populated primarily with zeros. A nonlocal means algorithm is developed to deal with these sparse coefficients to exploit the repetitive characters of structures in the whole image, realizing a "truly" global denoising. With synthetic and clinical data of ultrasound images and magnetic resonance images, this approach has been compared with other five state-of-the-art denoising methods. The experiments quantitative results demonstrate the effectiveness of our approach, especially superior in reducing the noise while well preserving the tissue details. It is concluded that our proposed approach is capable of enhancing image quality in both ultrasound and magnetic resonance images. It has a broad field of applications and will increase the diagnostic potential of the medical images.
\end{abstract}

Key words: Global denoising, medical images, patches' similarity, sparse representation.

\section{Introduction}

Due to the non-invasive, harmless and accurate nature, ultrasound imaging (US) and magnetic resonance imaging (MRI) are widely used in diagnoses and treatments [1]. However, the imaging acquisition and systems may introduce noises and artifacts, degrading contrast resolution and making medical images difficult for non-specialists to interpret. Thus, to achieve the best diagnosis, it is essential to denoise medical images without affecting important image features and destroying anatomical details [2].

In the past few decades, considerable efforts have been made in denoising the medical images. The local statistics methods form an output by a weighted average calculation using sub-region statistics over different pixel windows [3]-[5]. The median filter is a nonlinear operator, replacing the middle pixel in the window with the median value of its neighbours, effectively in eliminating single and unrepresentative pixels [6]. Anisotropic diffusion-based methods control the process of anisotropic diffusion based on statistical characteristics of noise [7], [8]. The parametric estimation methods like the maximum-likelihood filter and Bayesian denoising method establish noise models and adopt the robust parametric estimation approaches [9], [10]. The wavelet-based denoising algorithms based on multi-scale decompositions of the noisy images, apply soft thresholds to wavelet coefficients of different scales to eliminate the noise [11], [12]. Although the above filters are capable of effectively suppressing the speckle pattern, they still remove 
fine details. As the locally-based methods, they compromise between the averaging (in homogeneous regions) and preserving (at edges and features). Small window sizes or coefficients will retain the noise whereas large ones lead to the loss of signal details.

Different from locally-based methods, the nonlocal means (NLM) denoising algorithm takes advantage of the high degree of redundancy in the image [13], [14]. A sparse 3D transform-domain collaborative filtering (BM3D) groups the similar 2D image fragments into 3D groups by block matching and uses collaborative transform-domain shrinkage to reduce the noise [15]. They perform well in Gaussian noise reduction and sharp edges preservation. But the approximation as Gaussian noise will result in a biased estimate. Meanwhile, it employs the weighted Euclidean distance to search for the similar patches, which is time-consuming and difficult to realize in real applications.

In recent years, there has been a growing interest in the study of the sparse representation. Using an overcomplete dictionary that contains prototype atoms, images can be described by sparse linear combinations of these atoms. The K-Singular Value Decomposition (K-SVD) algorithm is an iterative method for adapting dictionaries to achieve sparse representations [16]. Applications of the sparse representation include denoising, compression and regularization, encompassing a persuasive potential in these fields.

In this paper, we propose a novel global denoising approach based on sparse representation and the NLM for medical images. Our main contributions are: (1) through an overcomplete and non-orthogonal dictionary, the original noisy image can be decomposed into the sparsest coefficients matrix populated primarily with zeros. Such matrix indicates the nature of the original image, greatly decreasing the computational complexity; (2) the weighted Euclidean distances in NLM are replaced by the sparse coefficients to exploit similar patterns in the whole image, which helps to achieve the "truly" global denoising; (3) the intensities of pixels in all similar small patches are used in the globally denoising. Unlike the general pixel-filtering, all pixels in a small patch are denoised simultaneously, accelerating the computation time.

\section{Methods}

\subsection{The K-SVD Decomposition for the Medical Images}

The K- SVD algorithm is one typical method for the sparse signal representation [16], [17]. Here, we focus the algorithm with the specific intent of decomposing the medical images into the sparsest coefficients. Through an overcomplete dictionary, the original image is decomposed into a sparse coefficients matrix populated primarily with zeros. Only several non-zero coefficients reveal the nature of the image, which greatly reduces the complexity of the original image.

For a noisy image $Y \in R^{M \times N}$, we consider to divide $Y$ into $L$ overlapping image patches of size $b \times b, L=$ $(M-b+1) \times(N-b+1)$. The image patches are ordered lexicographically as vectors $y_{i} \in R^{b^{2}}$. A sparse model defines an overcomplete dictionary matrix $D \in R^{b^{2} \times K}$ containing $K$ prototype atoms for columns $\left\{d_{j}\right\}_{j=1}^{K}$.

Then the image $Y$ can be represented as a sparse linear combination of these atoms. The K-SVD is designed to seek the sparsest representation coefficients $X$ that give the best image representation [27],

$$
\min _{X}\|Y-D X\|_{2}^{2} \quad \text { s.t. }\|X\|_{0} \leq T_{0}
$$

where $\|.\|_{0}$ is the ${ }^{0}$-norm, counting non-zero coefficients, $T_{0}$ is a sparsity threshold. According to (1), the sparse representation of $L$ image patches is:

$$
\min _{x_{i}}\left\|y_{i}-D x_{i}\right\|_{2}^{2} \quad \text { s.t. }\left\|x_{i}\right\|_{0} \leq T_{0} \quad i \in 1,2, \ldots L
$$


The goal is to find the dictionary $D$ that yields the sparest representation $\|X\|_{0}$ for the noisy image $Y$. Exact determination of sparse representation proves to be an NP-hard problem. Approximate solutions are considered instead. The K-SVD optimizes $D$ and $X$ through a number of iterations. Each iteration consists of two steps: (1) a sparse coding stage optimizing the coefficients $X$; (2) a dictionary updating stage improving the atoms in $D$. Recent development in theory reveals that if the solution $X$ is sparse enough, the solution of ${ }^{0}$-minimization problem is equivalent to pursuit problem. Here, an orthogonal matching pursuit (OMP) method is used to find the approximation one [16].

\subsection{Global Approach Based on the Image Patches' Similarity}

The locally-based image denoising methods use the "neighbourhood window" for filtering. The filtering effect and the edge preservation is a contradiction. As illustrated in Fig. 1, for a certain small patch $p_{1}$, if it is predicted by the non-relevant patches in its neighbourhood $S$, the fine structures and edges are smoothed out. However, $p_{1}$ may be well denoised by all similar patches $p_{2}, p_{3}, p_{4}$ and $p_{5}$, capable of taking care of the details. So we extend the "neighbourhood" to the "whole image". The redundancy of the texture images allows us to find many similar configurations in far away areas. The non-local methods greatly improve the denoising performance, especially in the edge preservation.

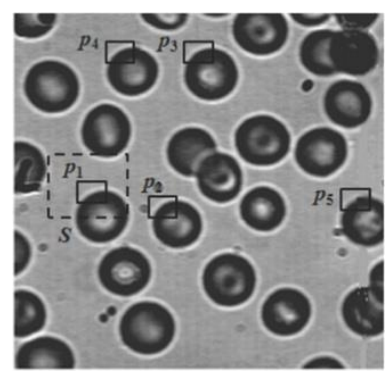

Fig. 1. Similar patches in the whole image.

How to find the similar small patches in the whole image is the key point in non-local methods. Several methods have been proposed to estimate the patches' similarity, including the mean, the variance, gray level co-occurrence matrix (GLCM), Euclidean distance and block matching [13], [18]. These methods are based on the pixel intensities, comparing the statistical characters or intensities between two patches. Due to the great complexity, it is hardly to realize. Hence, in the traditional NLM, it restricts the "search area" to a large window $\Omega$ with the size of $R_{\text {search }} \times R_{\text {search }}$ to reduce the operation time. Usually, $R_{\text {search }}=21$. The large search window $\Omega$ is more appropriate for the denoising and edge preserving. But it doesn't make full use of the redundancy in the whole image, ignoring the far-away similar patches.

The K-SVD decomposition makes the overcomplete dictionary $D$ containing many atoms, such as the atoms for the texture area and the atoms for the homogeneous area. The sparse coefficients of these two areas vary greatly. For the texture area, their coefficients involve a small number of non-zeros, while those of the homogeneous areas are all zeros. Thus, for computational purpose, the sum of each sparse coefficients vector, as $X_{-}$sum $\in R^{1 \times L}$, is acquired to estimate the patches' similarity.

$$
X_{-} \operatorname{sum}_{i}=\sum_{j=1}^{b^{2}} X_{j i} \quad i=1 \ldots L
$$

For the patch $y_{i}$ to be filtered, its differential vector $\Delta(i)$ can be obtained by subtracting its $X_{-}$sum ${ }_{i}$ from other patches' $X_{-}$sum $_{j}$. 


$$
\Delta(i)=\mid X_{-} \text {sum }_{i}-X_{-} \operatorname{sum}_{j} \mid \quad j=1 \ldots L(j \neq i)
$$

Sort $\Delta(i)$ in ascending order, the smaller $\Delta(i)$, the closer two patches. Choose the first $t$ columns, their corresponding image patches in the original image are considered to be the candidates.

To avoid the two patches with opposite characteristics having the same sum of sparse coefficients, the average intensity is employed to discard the irrelevant patches in the candidates set. For the $t$ candidates, calculating their mean intensity of the patches in the original image $Y$, named as $Y_{-}$mean $\in R^{1 \times L}$. If the patch $y_{k}$ close to the patch $y_{i}$, that is $\lambda_{1} \leq Y_{-}$mean $_{k} / Y_{-}$mean $_{i} \leq \lambda_{2}$, the patch $y_{k}$ is considered, where $\lambda_{1}<1$ and $\lambda_{2}>1$ are two constants close to one. Otherwise, the patch $y_{k}$ will be eliminated.

Therefore, the $n(n<t)$ most similar patches are saved, as $Y_{-}$similar $\in R^{b^{2} \times n}$. For further speed up, we set the same weights to each candidate. Unlike the general pixel filtering, here, we denoise all the pixels $(b \times b)$ in one small patch together. The denoised $\tilde{y}_{i}$ is defined as:

$$
\tilde{y}_{i}=\sum_{i=1}^{n} Y_{\text {similari }}
$$

$\tilde{y}_{i}$ is reshaped from column vector of the size $b^{2} \times 1$ to matrix of the size $b \times b$. For the overlapping pixels, we average their outcomes.

Our global denoising approach is as follows:

- A noisy image $Y$ is divided into $L$ overlapping image patches of size $b \times b$;

- $\quad Y$ is decomposed into a sparse coefficient matrix $X$ by K-SVD;

- $\quad$ Compute the sum of each sparse coefficients vector, $X \_s u m$;

- Calculate the differential vector $\Delta(\cdot)$;

- Sort $\Delta(\cdot)$ in ascending order and choose the first $t$ columns as the candidates;

- The intensity average matrix is further employed to discard the irrelevant patches in the candidate set;

- Estimate and reshape the denoised image $\tilde{y}_{i}$.

\section{Experiments and Results}

In this section, we carried out the experiments in two scenarios: US images (Rayleigh distributed) and MR images (Rician distributed). The performance of our approach was compared with other well-known methods in US and MR images denoising. The methods considered in this study were: the K-SVD [16], the NLM [13], the SRAD [8], the Portilla's wavelet [11] and the BM3D [15]. We chose the iterative number of the K-SVD as 10 and that of the SRAD as 150 to get the best results.

For the visual and quantitative evaluation, four criteria, including the visual display, the $S N R$, the mean structure similarity (MSSIM) and the figure of merit (FOM) were taken into account [8], [19]. The SNR was defined as,

$$
S N R(d B)=10 \lg \left[\sum_{i=1}^{M} \sum_{j=1}^{N} \frac{Y^{2}(i, j)}{\sum_{i=1}^{M} \sum_{j=1}^{N}(Y(i, j)-f(i, j))^{2}}\right]
$$

The higher the $S N R$ was, the less noise was.

The structure similarity (SSIM) was a method for measuring the similarity between two images [8]. It explored the structure information as an alternative motivating principle for the image quality measure. 
The SSIM was defined as,

$$
\operatorname{SSIM}(Y, f)=\frac{\left(2 \mu_{Y} \mu_{f}+C_{1}\right)\left(2 \sigma_{Y f}+C_{2}\right)}{\left(\mu_{Y}^{2}+\mu_{f}^{2}+C_{1}\right)\left(\sigma_{Y}^{2}+\sigma_{f}^{2}+C_{2}\right)}
$$

where $\mu_{g}, \mu_{f}, \sigma_{g}, \sigma_{f}, \sigma_{g f}$ denote the mean, variance and covariance of $8 \times 8$ square window which moved pixel-by-pixel in $Y(\cdot)$ and $f(\cdot)$ respectively. $C_{1}=K_{1} \times L, C_{2}=K_{2} \times L . L=255, K_{1}=0.01, K_{2}=0.03$. As the $S S I M$ was calculated on various windows of an image, we used the mean SSIM (MSSIM) to evaluate the overall image quality,

$$
\operatorname{MSSIM}(Y, f)=(1 / M) \times \sum_{i=1}^{M} \operatorname{SSIM}\left(Y_{i}, f_{i}\right)
$$

where $M$ was the number of local windows in the image. MSSIM $\in[0,1]$, the higher the $M S S I M$ was, the better the reservation of the texture was.

The figure of merit (FOM) was adopted to compare edge preservation performances [19]. The FOM was defined by,

$$
F O M=\left(1 / \max \left\{N, N_{\text {ideal }}\right\}\right) \times \sum_{i=1}^{N}\left(1 /\left(1+d_{i}^{2} \alpha\right)\right)
$$

where $N$ and $N_{\text {ideal }}$ were the number of the filtered and ideal edge pixels. $d_{i}$ was the Euclidean distance between ith filtered edge pixel and the nearest ideal edge pixel. $\alpha=1 / 9$. FOM $\in[0,1]$, with unity for ideal edge detection.

The parameters of the traditional NML method were optimized by a set of experiments in [18]. Two parameters: $b$ (the size of the small image patches) and $t$ (the number of the patch candidates) were set as $b=11$ and $t=80$.

\subsection{Denoising the Synthetic Images}

To assess the performance of the above mentioned methods, we performed experiments in two sets of the synthetic images. In the first scenario the image was simulated as US images, corrupted with speckle (Rayleigh distributed), in the second as MR images with Rician distributed noise. For the US synthetic images, the noisy images with different SNRs were generated by the original image multiplied with different Rayleigh-distributed noise levels. The parameter $\sigma_{\eta}^{2}$ varied in the interval $\{0.5,0.793,1,1.2,1.5,1.7,1.98$, $2.2,2.5,2.78,3.15,4,4.4,5\}$. For the MR synthetic images, a set of noise levels were added as the variance of Gaussian noise $\sigma_{n}^{2}=\{4,4.7,5.3,6,6.3,7.3,8.5,9.5,10.8,12,13,15\}$. Fig. 2 showed one example of the US synthetic images and the MR synthetic images respectively.

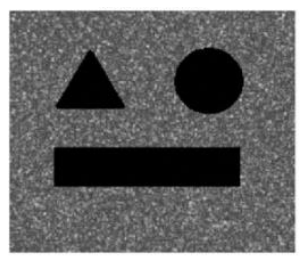

(a)

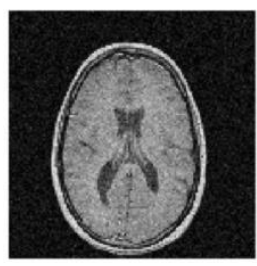

(b)

Fig. 2. The synthetic images, (a) the US image, (b) the MR image. 
Fig. 3, Fig. 4 and Table 1 were the results of different filters in the US synthetic images. As an example, Fig. 3 was the output of various methods at $S N R=11.45 \mathrm{~dB}$. Fig. 4 (a) and (b) were the MSSIM and FOM values of different $S N R$ s. Table 1 was the SNR comparison under different noise conditions.

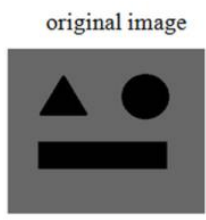

(a)

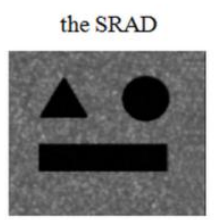

(e)

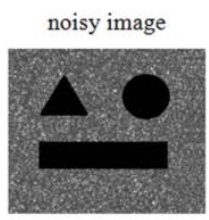

(b)

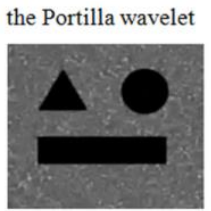

(f)

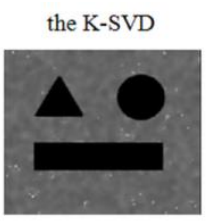

(c)

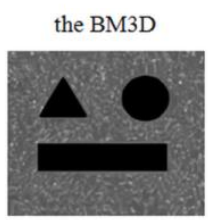

(g)

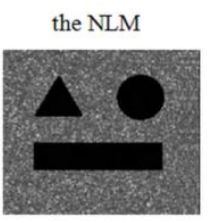

(d)

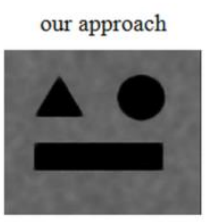

(h)

Fig. 3. The visual display of results of various filters in the US synthetic image at $S N R=11.45 \mathrm{~dB}$, (a) the original image, (b) the noisy image, (c)-(h) represents outputs of the filters labelled above the image.

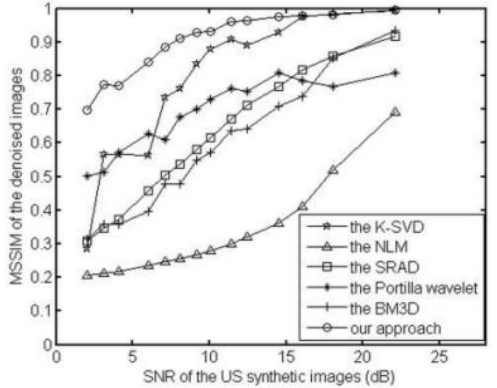

(a)

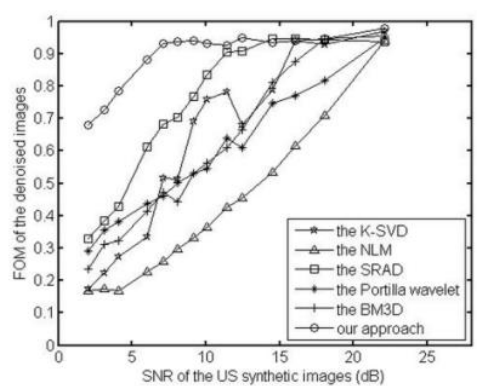

(b)

Fig. 4. The quantitative comparisons of the US synthetic images, (a) the MSSIM, (b) the FOM.

Table 1. The SNR Comparison under Different Noise Conditions for the US Images (dB)

\begin{tabular}{ccccccccccccccc}
\hline method & 2.02 & 3.12 & 4.11 & 5.99 & 7.14 & 8.02 & 9.14 & 10.1 & 11.4 & 12.5 & 14.5 & 16.0 & 18.1 & 22.1 \\
\hline the K-SVD & 7.43 & 12.5 & 12.9 & 13.8 & 16.7 & 17.6 & 19.9 & 21.7 & 23.1 & 22.6 & 25.0 & 28.8 & 30.2 & 35.1 \\
\hline the NLM & 2.01 & 3.16 & 3.90 & 6.14 & 7.23 & 8.14 & 9.17 & 10.1 & 11.4 & 12.5 & 14.4 & 16.0 & 18.7 & 23.2 \\
\hline the SRAD & 9.54 & 11.0 & 11.9 & 14.2 & 15.4 & 16.3 & 16.9 & 17.9 & 18.8 & 19.7 & 20.8 & 21.9 & 22.8 & 24.0 \\
\hline $\begin{array}{c}\text { the Portilla } \\
\text { wavelet }\end{array}$ & 13.1 & 14.2 & 14.4 & 15.2 & 15.8 & 17.2 & 18.1 & 18.6 & 19.6 & 19.8 & 21.2 & 21.4 & 22.6 & 24.7 \\
\hline $\begin{array}{c}\text { The BM3D } \\
\begin{array}{c}\text { our } \\
\text { approach }\end{array}\end{array}$ & 15.92 & 10.4 & 10.8 & 12.3 & 14.3 & 14.4 & 16.2 & 17.2 & 18.4 & 18.8 & 20.9 & 22.1 & 25.1 & 29.4 \\
\hline \hline
\end{tabular}

Fig. 5, Fig. 6 and Table 2 were the results of different filters in the MR synthetic images. As an example, Fig. 5 was the output of various methods at $S N R=11.02$ dB. Fig. 6 (a) and (b) were their MSSIMs and FOMs. Table 2 was the SNR comparison.

In Fig. 3, we observed that the NLM, the BM3D, the K-SVD and the Portilla's wavelet worked well with the Gaussian noise, but failed in the Rayleigh noise. The speckle was clearly visible in the background. The SRAD was a classic method in the US image denoising. But it also remained noise the homogeneous area, 
with the image blurred. Apparently, our proposed method had the best despeckling effect, with the highest MSSIM and FOM values. Table 1 corroborated with the visual observation. Compared with the other filter, our approach improved the SNRs of the noisy images greatly, especially superior for the high-noise levels.

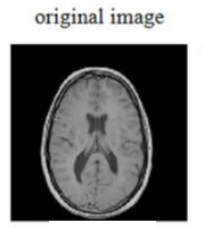

(a)

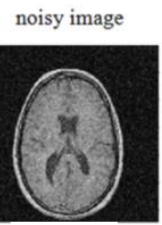

(b)

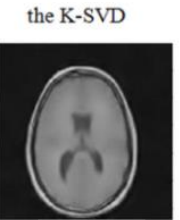

(c) the NLM

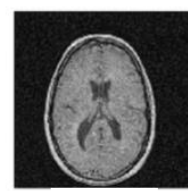

(d)

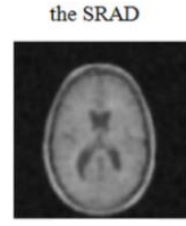

(e)

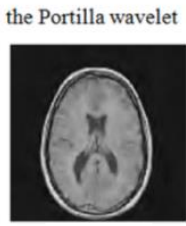

(f)

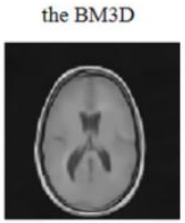

(g)

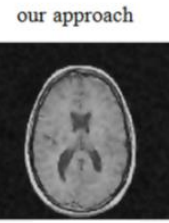

(h)

Fig. 5. The visual display of results of various filters in the MR synthetic image at $S N R=11.02 \mathrm{~dB}$, (a) the original image, (b) the noisy image, (c)-(h) represents outputs of the filters labelled above the image.

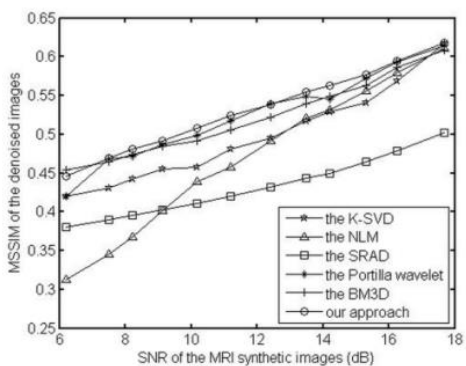

(a)

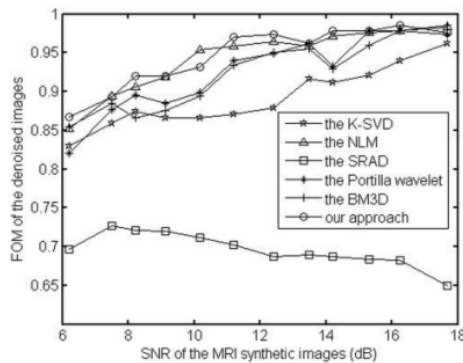

(b)

Fig. 6. The quantitative comparisons of the MR synthetic images, (a) the MSSIM, (b) the FOM.

Table 2. The SNR Comparison under Different Noise Conditions for the MR Images (dB)

\begin{tabular}{|c|c|c|c|c|c|c|c|c|c|c|c|c|}
\hline method & 6.20 & 7.49 & 8.23 & 9.13 & 10.2 & 11.2 & 12.4 & 13.5 & 14.2 & 15.3 & 16.2 & 17.7 \\
\hline the K-SVD & 7.47 & 8.53 & 9.19 & 9.98 & 10.7 & 11.7 & 12.7 & 13.8 & 14.3 & 14.9 & 15.9 & 17.5 \\
\hline the NLM & 6.55 & 7.81 & 8.51 & 9.51 & 10.7 & 11.6 & 13.1 & 14.2 & 14.7 & 15.8 & 16.7 & 17.9 \\
\hline the SRAD & 6.92 & 7.81 & 8.19 & 8.75 & 9.32 & 9.85 & 10.4 & 10.9 & 11.1 & 11.4 & 11.6 & 11.9 \\
\hline $\begin{array}{c}\text { the Portilla } \\
\text { wavelet }\end{array}$ & 7.79 & 8.19 & 9.81 & 10.3 & 11.3 & 12.3 & 13.7 & 14.1 & 14.5 & 15.8 & 16.2 & 17.1 \\
\hline the BM3D & 7.75 & 9.01 & 9.57 & 10.6 & 11.4 & 12.4 & 13.6 & 14.4 & 14.9 & 16.0 & 16.5 & 17.0 \\
\hline our approach & 8.79 & 9.05 & 9.64 & 10.6 & 11.6 & 12.5 & 13.8 & 14.6 & 15.2 & 16.1 & 16.8 & 17.5 \\
\hline
\end{tabular}

It could be seen from Fig. 5 that both the K-SVD and the SRAD were over smoothed in MR images, with lots of tissue details lost. Since the MR images with Rician distribution could be well approximated by a Gaussian distribution at high SNRs. The methods with the assumption of the noise model being Gaussian showed good denoising performances. The NLM preserved more features, while had poor noise suppression ability. The BM3D improved the NLM in flat zone, but over-smoothed the contours and details. The Portilla's wavelet obscured the image. In Table 2, the SNRs of the different filters were not obvious. In 
the aspect of the MSSIM and the FOM, the proposed method was very competitive. It revealed that the edge preservation was the most remarkable merit of our denoising method.

\subsection{Denoising the Clinical Medical Images}

We applied the proposed methods to the clinical medical images, including the breast tumor US image, and the liver MR image, as shown in Fig. 7 and Fig. 8.

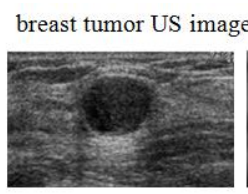

(a)

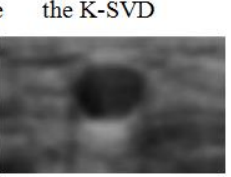

(b)

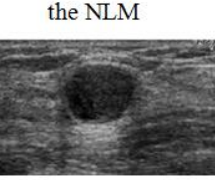

(c) the SRAD

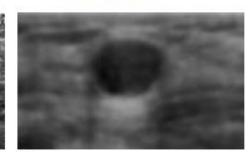

(d)

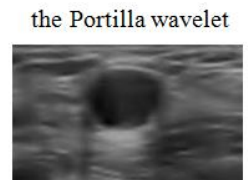

(e)

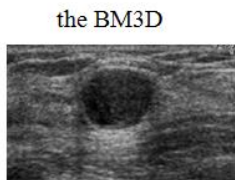

(f)

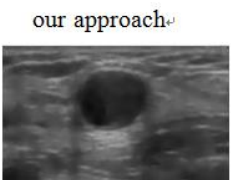

(g)

Fig. 7. Denoised results of a breast tumor US image, (a) the original image, (b)-(g) represents outputs of the filters labelled above the image.

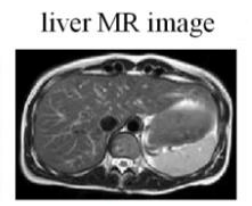

(a)

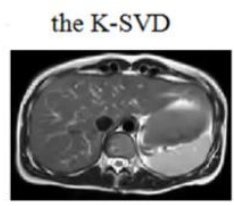

(b)

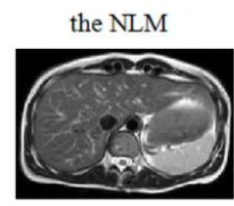

(c)

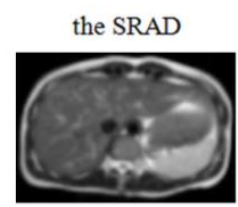

(d)

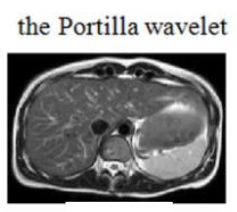

(e)

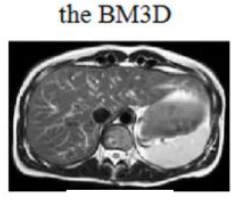

(f)

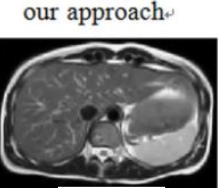

(g)

Fig. 8. Denoised results of a liver MR image, (a) the original image, (b)-(g) represents outputs of the filters labelled above the image.

In the US and MR imaging, it was seen that the NLM, the Portilla's wavelet and the BM3D were suitable for the MRI images, but failed in the despeckling. Compared with the originally US images, the speckle was still noticeable in both solutions. The SRAD provided the significant speckle suppression, with the cost of excessive smoothing details. The K-SVD blurred the output, with a lot of features lost. Our approach achieved the balance between the noise suppression and the feature reservation both for the US images and MR images. Some important imaging details were better preserved in the denoised image.

\section{Conclusion}

In this paper, a new and efficient technique for denoising the medical images has been proposed. We have designed a global denoising approach via the sparse representation for the Rayleigh distributed US images and the Rician distributed MR images. This method utilizes the sparse coefficients of the image, seeking the most similar patches in the whole image. Experimental results on the synthetic and clinical medical images are present to evaluate the performance of the proposed method. A comparison with the state of the art 
denoising methods demonstrated that our approach manages to achieve competitive results in a wide variety of the experimental conditions. It yields better noise attenuation and edge enhancement, especially works excellently in containing fine structures

\section{Acknowledgment}

This work was supported by the National Basic Research Program of China (No. 2015CB755500), the National Natural Science Foundation of China (Grant 61401102) and the Science and Technology Commission of Shanghai Municipality (Grant 14YF1400300).

\section{References}

[1] Manning, D., Gale, A., \& Krupinski, E. (2007). Perception research in medical imaging. British Journal of Radiolog, 78(932), 683-685.

[2] Sanches, J., Nascimento, J., \& Marque, J. (2008). Medical image noise reduction using the Sylvester-Lyapunov Equation. IEEE Transactions on Image Processing, 17(9), 1522-1539.

[3] Lee, J. (1981). Speckle analysis and smoothing of synthetic aperture radar images. Computer Graphics and Image Processing, 17(1), 24-32.

[4] Frost, V., Stiles, J., Shanmuggam, K., \& Holtzman, J. (1982). A model for radar images and its application for adaptive digital filtering of multiplicative noise. IEEE Transactions on Pattern Analysis and Machine Intelligence, 4(2), 157-165.

[5] Kuan, D., Sawchuk, A., Strand, T., \& Chavel, P. (1987). Adaptive restoration of images with speckle. IEEE Transactions on Acoustics Speech and Signal Processing, 35(3), 373-383.

[6] Vanithamani, R., Umanaheswari, G., \& Ethilarasi, M. (2010). Modified hybrid median filter for effective speckle reduction in ultrasound images. Proceedings of the 12th International Conference on Networking, VLSI and signal processing (pp.166-171).

[7] Deng, Y., Wang, Y., \& Shen, Y. (2011). Speckle reduction of ultrasound images based on Rayleigh-trimmed anisotropic diffusion filter. Pattern Recognition Letters, 32(13), 1516-1525.

[8] Yu, Y., \& Acton, S. (2002). Speckle reducing anisotropic diffusion. IEEE Transactions on Image Processing, 11(11), 1260-1270.

[9] Shankar, P. (2006). Speckle reduction in ultrasonic images through a maximum likelihood based adaptive filter. Physics in Medicine and Biology, 51(21), 5591-5602.

[10] Rajan, J., Jeurissen, B., Verhoye, M., Audekerke, J., \& Sijbers, J. (2011). Maximum likelihood estimation-based denoising of magnetic resonance images using restricted local neighbourhoods. Physics in Medicine and Biology, 56(16), 5221-5234.

[11] Portilla, J., Strela, V., Wainwright, M., \& Simoncelli, E. (2003). Image denoising using scale mixtures of Gaussians in the wavelet domain. IEEE Transactions on Image Processing, 12(11), 1338-1351.

[12] Taya, P., Acton, S., \& Hossack, J. (2011). A wavelet thresholding method to reduce ultrasound artifacts. Computerized Medical Imaging and Graphics, 35(1), 42-50.

[13] Buades, A., Coll, B., \& Morel, A. (2005). A review of image denoising algorithms with a new one. Multiscale Modeling \& Simulation, 4(2), 490-530.

[14] Manjón, J., Caballero, J., Lull, J., Mart, G., Bonmat, L., \& Robles, M. (2008). MRI denoising using non-local means. Medical Image Analysis, 12(4), 514-523.

[15] Dabov, K., Foi, A., Katkovnik, V., \& Egiazarian, K. (2007). Image denoising by sparse 3-D transform domain collaborative filtering. IEEE Transactions on Image Processing, 16(8), 2080-2095.

[16] Aharon, M., Elad, M., \& Bruckstein, A. (2006). K-SVD: An algorithm for designing overcomplete dictionaries for sparse representation. IEEE Transactions on Signal Processing, 54(11). 4311-4322. 
[17] Elad, M., Figueiredo, M., \& Ma, Y. (2010). On the role of sparse and redundant representations in image processing. Proceedings of the IEEE, 98(6), 972-982.

[18] Guo, Y., Wang, Y., \& Hou, T. (2011). Speckle filtering of ultrasonic images using a modified non local-based algorithm. Biomedical Signal Processing and Control, 6(2), 129-138.

[19] Wang, Z., Bovik, A., Sheikh, H., \& Simoncelli, E. (2004). Image quality assessment: From error visibility to structural similarity. IEEE Transactions on Image Processing, 13(4), 600-612.

Yi Guo received the B.Sc. degree in electronic engineering, and the M.Sc. and Ph.D. degrees in biomedical engineering from Fudan University, Shanghai, China, in 2013. She is now a lecturer in Department of Electronic Engineering in Fudan University. Her research interests include medical signal and image processing.

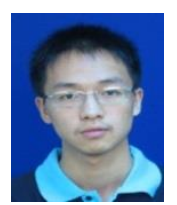

Hanchao Chai received the bachelor's degree in biomedical engineering from Fudan University, Shanghai China, in 2013.

He is now studying in Department of Electronic Engineering in Fudan University for the master' s degree. His research interest is in medical image segmentation.

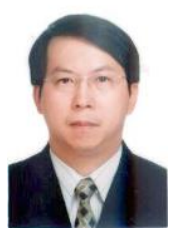

Yuanyuan Wang received the B.Sc., M.Sc. and Ph.D. degrees in electronic engineering from Fudan University, Shanghai, China, in 1990, 1992 and 1994, respectively. During 1994 to 1996, he was a postdoctoral research fellow with the school of electronic engineering and computer science at University of Wales, Bangor, UK. In May 1996, he went back to Department of Electronic Engineering at Fudan University as an associate professor. He was then promoted to a full professor in May, 1998. He is currently the director of biomedical engineering center and vice dean of Information Science and Engineering School at Fudan University. He is also the author or coauthor of 6 books and 430 research papers. His research interests include medical ultrasound techniques and medical signal processing. 